\title{
EULAR recommendations for the management and vaccination of people with rheumatic and musculoskeletal diseases in the context of SARS- CoV-2: the November 2021 update
}

\author{
Robert B M Landewé 다 , ${ }^{1,2}$ Féline P B Kroon (1) , ${ }^{3,4}$ Alessia Alunno (1) ,5

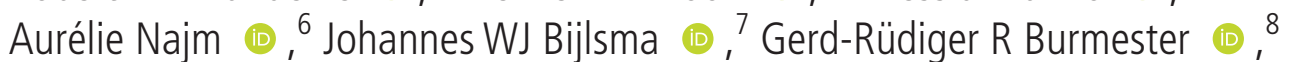 \\ Roberto Caporali, ${ }^{9}$ Bernard Combe, ${ }^{10}$ Richard Conway (1) , ${ }^{11}$ Jeffrey R Curtis, ${ }^{12}$ \\ Ori Elkayam, ${ }^{13}$ Laure Gossec $\odot$, ${ }^{14,15}$ Marloes W Heijstek, ${ }^{16}$ Lukas Haupt, $^{17}$ \\ Annamaria lagnocco $(1),{ }^{18}$ John D Isaacs $(1),{ }^{19}$ István Ábel Juhász, ${ }^{20}$ Suzi Makrii, ${ }^{21}$ \\ Xavier Mariette @ ${ }^{22,23}$ lain B McInnes, ${ }^{24,25}$ Puja Mehta (1), ${ }^{26,27}$ Ulf Mueller-Ladner, ${ }^{28}$ \\ Hendrik Schulze-Koops (1), ${ }^{29}$ Josef S Smolen, ${ }^{30}$ Dieter Wiek, ${ }^{31}$ Kevin L Winthrop (i) , ${ }^{32}$ \\ Victoria Navarro-Compán 다, 33 Pedro M Machado (i) 34,35,36
}

\section{Handling editor Francis Berenbaum \\ For numbered affiliations see end of article.}

\section{Correspondence to}

Professor Robert B M Landewé, Amsterdam UMC Locatie AMC, Amsterdam, North Holland, The Netherlands;

landewe@rlandewe.nl

Received 16 December 2021 Accepted 25 January 2022
Check for updates

(C) Author(s) (or their employer(s)) 2022. No commercial re-use. See rights and permissions. Published by BMJ.

To cite: Landewé RBM, Kroon FPB, Alunno A, et al. Ann Rheum Dis Epub ahead of print: [please include Day Month Year]. doi:10.1136/ annrheumdis-2021-222006

\section{ABSTRACT}

The first EULAR provisional recommendations on the management of rheumatic and musculoskeletal diseases (RMDs) in the context of severe acute respiratory syndrome coronavirus 2 (SARS-CoV-2), largely based on expert opinion, were published in June 2020. Since then, an unprecedented number of clinical studies have accrued in the literature. Several SARS-CoV-2 vaccines have been approved for population-wide vaccination programmes in EULAR-affiliated countries. Studies regarding vaccination of patients with (inflammatory) RMDs have released their first results or are underway. EULAR found it opportune to carefully review to what extent the initially consensus expert recommendations stood the test of time, by challenging them with the recently accumulated body of scientific evidence, and by incorporating evidence-based advice on SARS-CoV-2 vaccination. EULAR started a formal (first) update in January 2021, performed a systematic literature review according to EULAR's standard operating procedures and completed a set of updated overarching principles and recommendations in July 2021. Two points to consider were added in November 2021, because of recent developments pertaining to additional vaccination doses.

\section{INTRODUCTION}

EULAR's first set of provisional recommendations addressing several clinical aspects of severe acute respiratory syndrome coronavirus 2 (SARS-CoV-2) and the disease caused by SARS-CoV-2, coronavirus disease 2019 (COVID-19), was published in June 2020. ${ }^{1}$ The document addressed the implications of the pandemic for patients with rheumatic and musculoskeletal diseases (RMDs), at a time when very little was known about the epidemiology and the clinical course of patients with RMDs who contracted SARS-CoV-2 infection, and in particular about the risks that patients with RMDs faced, as well as preventive measures that these patients and their caregivers should take. The task force that dealt with the matter was-from a scientific point of view-flying blindly and had to rely on sparse clinical experience, a lot of common sense and a paucity of scientific evidence. Two factors may explain the delay in updating the first set of recommendations: (1) While the amount of data about SARS-CoV-2 infection/COVID-19 and RMDs in the literature accrued exponentially, the content of the original EULAR recommendations appeared to remain remarkably current, which in the opinion of the steering committee eliminated the urgency of an immediate update; (2) The advent of SARSCoV-2 vaccinations by the beginning of 2021 and the initiation of epidemiological vaccination studies in patients with RMDs prompted the steering group to decide to issue an ad hoc advice on vaccination of patients with RMDs in December $2020^{2}$ and to postpone a formal systematic literature review (SLR) until more comprehensive studies had been published.

Finally, EULAR decided to start the update process in January 2021, with a formal two-tier SLR, one covering the preceding year with a deadline of 29 March 2021 and the other covering the remaining months with a deadline of 31 May 2021. The formal SLR was expanded by a post hoc search for additional vaccination studies, on the request of the reviewers of the SLR manuscript, with a deadline of 11 October 2021.

As stated previously, ${ }^{1}$ EULAR does not intend to over-rule existing guidelines at the country-level of EULAR member states. EULAR aims to provide a synthesis of the best available evidence ('the SLR') and the aggregated expert opinion, to inform rheumatologists and other healthcare providers (HCPs), as well as patients with RMDs about management decisions to be taken in the context of the global pandemic.

Unlike the unprecedented circumstances and urgency at the beginning of the pandemic, during which the provisional recommendations had to be 
developed, the task force has now carefully followed the standard operating procedures (SOPs) ${ }^{3}$ for updating management recommendations. As before, the task force was limited by restrictions of social distancing, preventing them from meeting in person and the entire process was conducted remotely by videoconferencing.

\section{PROCEDURES}

\section{Focus of recommendations}

These recommendations pertain to the management of patients with RMDs as the SARS-CoV-2 pandemic and its consequent COVID-19 disease may interfere with their usual management. The recommendations do not focus on diagnosing or treating COVID-19.

Most focus is on 'inflammatory' RMDs, because most questions from HCPs and patients themselves pertained to systemic autoimmune diseases, in particular to their treatments, as well as to the risks and benefits of vaccination against SARS-CoV-2. Needless to say that these recommendations also include patients with 'non-inflammatory' RMDs.

\section{The task force composition}

This EULAR task force consists of 28 members, 26 from 11 EULAR member states and 2 from the USA. Many expert members are internationally recognised rheumatologists and immunologists with many years of clinical and scientific experience, who fulfil or have fulfilled official positions in the EULAR organisation. EULAR's current and past presidents (AI, GRB, IBM, JSS and JWJB), as well as the current chair of EULAR's committee for the quality of care (RBML), the current chair of EULAR's people with arthritis and rheumatism (PARE) committee (SM) and EULAR's past vice-president representing PARE (DW) are members of the task force, among others. Five seats in the task force were reserved for rheumatologists from EULAR countries who could apply for this position and were subsequently selected by the convenor (RBML). Two seats were reserved for members of the emerging EULAR network (EMEUNET) who could apply for this position and were selected by the EMEUNET steering committee (PM and RC). The task force was further completed by an expert in infectious diseases (KW), one nominated representative of the American College of Rheumatology (ACR) (JC), three SLR fellows (FPBK, AA and AN), one senior methodologist and past-chair of the standing committee on epidemiology and health services research (PMM) and one junior methodologist (VN-C). The steering committee was formed by the convenor (RBML), the two methodologists (PMM and $\mathrm{VN}-\mathrm{C}$ ) and the three fellows (FPBK, AA and AN). All taskforce members were informed about, or had prior experience of, the development of EULAR recommendations according to EULAR's SOPs. ${ }^{3}$

\section{Handling potential conflict of interest}

In accordance with EULAR's SOPs, task force members are asked on an annual basis to provide and update their interactions with third parties (guideline committees, reimbursement bodies, pharmaceutical industries or other industries) that are not directly related to daily patient care but may give an impression to others of conflict of interest (potential COI). The EULAR office keeps record of these declared potential COIs.

\section{The steering committee's workflow and procedures}

The steering committee convened several times by videoconference and prepared the task force meetings and the SLR, as well as the draft updates of overarching principles (OPs) and recommendations, all for discussion and decision-making among the entire task force. The steering committee, in particular the convenor and methodologists, supervised the fellows' SLR work, discussed the application of instruments for risk of bias assessment, performed together with the fellows the actual risk of bias assessment and approved the reports of the SLR for dissemination among the task force members. Finally, the steering committee solicited the levels of agreement from task force members (by anonymous online survey), determined levels of evidence per item (according to the 2011 Oxford Centre for Evidence-Based Medicine) and drafted (two) manuscripts that were submitted to the EULAR Council for formal approval.

\section{The task force's workflow and procedures}

The task force members reviewed the preparatory work sent to them by email and were given the opportunity to propose changes. The task force convened by videoconference in four separate sessions: the first on 19 January 2021, in which the research questions for SLR were established; a second meeting on 25 May 2021, in which the task force was informed about the results of the first tier of the SLR; a third meeting on 16 July 2021 , in which the task force was informed about the results of the second tier of the SLR and in which consensus about updated OPs and recommendations was reached; and a fourth meeting on 16 November 2021, in which the task force was informed about the results of the post hoc SLR limited to vaccination studies, and in which consensus was reached about two additional points to consider pertaining to additional vaccination doses. All task force members reviewed, discussed and agreed to the final version of this manuscript before submission to the EULAR Council.

\section{Target audience}

In line with EULAR's SOPs, the task force agreed to target their guidance primarily for rheumatologists, and other HCPs, and for patients with RMDs and their families. Secondarily, these recommendations target public health officials and public health experts by making them aware of particular problems pertaining to patients with RMDs and their treatments, as well as policymakers, who decide about infection prevention and control measures, access to healthcare for patients with RMDs, SARSCoV-2 vaccination and availability of drugs for patients with RMDs.

\section{Systematic literature research}

The procedures, course and results of the SLR are described in detail in an accompanying article. ${ }^{4}$

\section{Formal decision-making}

Formal voting was only performed when deemed necessary during the task force meeting on 16 July 2021. Questions for voting were formulated by the meeting chair (RBML) in such a manner that a choice between two options (A and B) remained, and voting took place using the chat function of Microsoft Teams virtual platform. Voting was not blind, results were aggregated by non-voting EULAR staff present at the meeting and EULAR voting rules for making decisions applied (consensus accepted if $>75 \%$ of the members voted in favour of the recommendation at the first round, $\geq 67 \%$ at the second round and at a third round $>50 \%$ was accepted). If thresholds were not met, unresolved questions were rediscussed and the voting question was reformulated for subsequent voting. This process was repeated until a formal decision was reached. Each expert's level of 
Table 1 EULAR recommendations for the management of rheumatic and musculoskeletal diseases in the context of SARS-CoV-2: the November 2021 update

\begin{tabular}{|c|c|c|c|}
\hline & Overarching principles & LoA, mean (SD) & $\% \geq 8 / 10$ \\
\hline 1. & In general, patients with RMDs do not face higher risk of contracting SARS-CoV-2 than individuals without RMDs, and do not have a worse prognosis when they contract it. & $8.8(1.5)$ & 81 \\
\hline 2. & The diagnosis and treatment of COVID-19 in patients with RMDs is the primary responsibility of an expert in treating COVID-19. & $9.9(0.3)$ & 100 \\
\hline 3. & $\begin{array}{l}\text { Rheumatologists are the leading experts for the immunomodulatory or immunosuppressive treatments of their patients and should be involved in the decision to maintain or } \\
\text { discontinue them. }\end{array}$ & $9.9(0.4)$ & 100 \\
\hline 4. & In view of their expertise, rheumatologists should be engaged in local hospital, regional or national guideline committees for COVID-19 management. & $9.2(1.2)$ & 89 \\
\hline \multirow[t]{2}{*}{5.} & $\begin{array}{l}\text { The off-label use of immunomodulatory or immunosuppressive drugs for the treatment of COVID-19 outside of established guidelines, protocols or clinical trials should be } \\
\text { discouraged. }\end{array}$ & $9.2(1.2)$ & 93 \\
\hline & Recommendations & & \\
\hline 1. & $\begin{array}{l}\text { Patients with RMDs should be strongly advised to comply with all infection prevention and control measures prescribed by public health authorities, before and after SARS- } \\
\text { CoV-2 vaccination. }\end{array}$ & $9.9(0.2)$ & 100 \\
\hline 2. & Patients with RMDs should be advised to receive SARS-CoV-2 vaccination with any of the vaccines approved in their country. & $9.6(1.6)$ & 96 \\
\hline 3. & $\begin{array}{l}\text { Patients with RMDs who have been vaccinated against SARS-CoV-2 should be advised to continue their treatment unchanged; those who have not been vaccinated should be } \\
\text { advised to continue their treatment, taking into account that certain therapies have been associated with an increased risk of severe COVID-19. }\end{array}$ & $9.5(0.6)$ & 100 \\
\hline 4. & If a patient with RMD receiving long-term glucocorticoid treatment develops suspected or confirmed COVID-19, this treatment should be continued. & $9.3(0.9)$ & 96 \\
\hline 5. & If a patient with RMD receiving rituximab treatment contracts SARS-CoV-2, postponing the next cycle of rituximab should be considered. & $9.7(0.6)$ & 100 \\
\hline 6. & $\begin{array}{l}\text { Patients with RMDs and initially mild symptoms who experience worsening of COVID-19 symptoms should immediately seek further healthcare advice of an expert in treating } \\
\text { COVID-19. }\end{array}$ & $9.9(0.3)$ & 100 \\
\hline 7. & $\begin{array}{l}\text { Patients with RMDs should be advised to update their general vaccination status in accordance with the EULAR recommendations for the vaccination of patients with RMDs, } \\
\text { with a particular focus on pneumococci and influenza. }\end{array}$ & $9.7(0.6)$ & 100 \\
\hline 8. & $\begin{array}{l}\text { In patients with RMDs not using immunomodulatory or immunosuppressive treatment, SARS-CoV-2 vaccination should precede a treatment start with such therapy if clinically } \\
\text { feasible. }\end{array}$ & $9.6(1.1)$ & 93 \\
\hline \multirow[t]{2}{*}{9.} & In patients with RMDs using rituximab or another B-cell depleting therapy, SARS-CoV-2 vaccination should be scheduled in a way to optimise vaccine immunogenicity. & $9.6(1.1)$ & 96 \\
\hline & Points to consider & & \\
\hline 1. & $\begin{array}{l}\text { There are concerns that individuals on certain immunosuppressive or immunomodulatory drugs may not mount an adequate protective response to COVID-19 vaccination. Data } \\
\text { are not currently available to reliably identify who might, or might not, benefit from a third primary dose of a SARS-CoV-2 vaccine. Taking a precautionary position, third primary } \\
\text { doses are being recommended by some authorities in selected groups of individuals and EULAR supports this approach. }\end{array}$ & $9.7(0.6)$ & 100 \\
\hline 2. & $\begin{array}{l}\text { There are concerns that protection provided by vaccines against severe COVID-19 decreases gradually over time. Insufficient time has passed to know what levels of protection } \\
\text { might be expected } 4-6 \text { months after the primary course. Taking a precautionary position, booster doses are being recommended by several authorities and EULAR supports this } \\
\text { approach. }\end{array}$ & $9.4(1.0)$ & 95 \\
\hline
\end{tabular}

agreement (from 0 (no agreement at all) to 10 (full agreement)) with the statement was solicited after the final task force meeting by anonymous online survey for each OP and recommendation. The mean level of agreement, as well as the proportion of experts with a level of agreement of at least 8 , was calculated.

\section{RESULTS}

The previous version of the recommendations contained 5 OP and 13 recommendations. ${ }^{1}$ In the update process, the task force agreed on 5 OP, 9 recommendations and 2 additional points to consider (table 1). The bullet-text of these OP, recommendations and points to consider can be read in table 1. Below, an item-byitem discussion serves to give insight into the reasoning of task force members, focuses on how previous items and new items relate to each other and provides a justification for amendments and additions.

Old OP 1: To date, there is no evidence that patients with RMDs face more risk of contracting SARS-CoV-2 than individuals without RMDs, nor that they have a worse prognosis when they contract it.

New OP 1: In general, patients with RMDs do not face more risk of contracting SARS-CoV-2 than individuals without RMDs, and do not have a worse prognosis when they contract it.

New OP 1 is almost unchanged, but its evidence base has considerably improved, as the results of the SLR demonstrate. While the old OP 1 was preceded by the words To date, in order to reflect the scarcity of reliable data, many studies have been published thereafter and testify to the credibility of the statement. This statement pertains to the incidence of COVID-19 among patients with RMDs, as well as to the risk factors for contracting COVID-19 and for an unfavourable clinical course of COVID-19: while patients with RMDs may generally face worse outcomes and increased mortality, the incidence, risk and course of COVID-19 are globally the same as in the general population.

The words In general have been added to the new OP 1 to refer to a few situations in which the accuracy of the global statement can be disputed. Examples are patients with some rare and severe systemic autoimmune or autoinflammatory diseases. ${ }^{4}$ Obviously, as a consequence of their scarcity, these exceptional cases have not yet been studied well. The same reservation pertains to certain treatments that have been associated with a worse COVID-19 course, such as rituximab, mycophenolic acid/mycophenolate mofetil (MMF), glucocorticoids (discussed under new recommendation (RC) 4) and potentially Janus kinase inhibitors (JAKi) (discussed under new RC 3). ${ }^{5-9}$ The taskforce discussed that either methodological considerations preclude a firm(er) stand, or that the drug in question was too infrequently investigated in studies to base a general statement on. While these examples are more explicitly addressed in the SLR for reference, ${ }^{4}$ they were kept out of the realm of the OP and recommendations (the exception to the rule being rituximab, as further outlined below).

Level of agreement: 8.8/10.

Old OP 2: The diagnosis and treatment of COVID-19 in patients with RMDs is the primary responsibility of an expert in treating COVID-19, such as a pulmonologist, an internist or a specialist in infectious diseases, dependent on local circumstances.

New OP 2: The diagnosis and treatment of COVID-19 in patients with RMDs is the primary responsibility of an expert in treating COVID-19.

This OP did not change significantly. It was considered more clear now than it was in the past that other medical experts than rheumatologists are primarily responsible for the treatment of 
COVID-19. The task force felt that further specification of those experts was redundant and beyond the scope of this task force, especially since the situation may vary per country, per region and per hospital.

Level of agreement: 9.9/10.

Old OP 3: Rheumatologists are the leading experts for the immunosuppressive treatments of their patients and should be involved in the decision to maintain or discontinue them.

New OP 3: Rheumatologists are the leading experts for the immunomodulatory or immunosuppressive treatments of their patients and should be involved in the decision to maintain or discontinue them.

While this OP has not substantially changed, the term 'immunomodulatory or immunosuppressive treatment' is introduced here for the first time, and will be used throughout the entire document. Already from the beginning (in April 2020) there was dissent about using the term 'immunosuppressive' versus 'immunomodulatory', which led to an explanatory Viewpoint by Isaacs and Burmester, ${ }^{10}$ who argued that some of the drugs used in rheumatology are 'immunomodulatory' (eg, targeted therapies), while others are 'immunosuppressive' (eg, glucocorticoids, azathioprine and MMF), and that the 'immunosuppressive' designation should not be used to cover all these drugs. Therefore, the task force decided to use the terminology 'immunomodulatory or immunosuppressive' throughout the document.

Level of agreement: 9.9/10.

Old OP 4: The knowledge about immunosuppressive treatments, including synthetic DMARDs and biological DMARDs, for the treatment of severe COVID-19 is rapidly evolving. In view of their expertise, rheumatologists should make themselves available for local-hospital, regional or national guideline committees for COVID-19. The use of immunosuppressive drugs for the treatment of COVID-19 should be a multidisciplinary decision.

New OP 4: In view of their expertise, rheumatologists should be engaged in local-hospital, regional or national guideline committees for COVID-19 management.

This OP has been condensed by virtue of evolving evidence. During the pandemic it has become clear that some of the treatments often used by rheumatologists have gained a prominent position in the management of patients with a hyperinflammatory state due to COVID-19 (eg, Kineret and tocilizumab, recently approved by the European Medicines Agency (EMA) for the treatment of adults with severe COVID-19 who are receiving systemic treatment with corticosteroids and require supplemental oxygen or mechanical ventilation), since randomised controlled trials (RCTs) have proven their efficacy and several are currently under review for marketing authorisation by the EMA, while a drug like hydroxychloroquine, promoted as a potentially lifesaving compound in the beginning of the pandemic, has clearly been discredited after the results of several RCTs were published. Given that rheumatologists are the experts with the most experience in the benefits and risks, pharmacokinetics and pharmacodynamics of glucocorticoids and targeted therapies, such as interleukin-6-receptor (IL-6R) inhibitors and JAKi, rheumatologists are well placed to be involved in guideline developments that include such treatments for COVID-19.

Level of agreement: 9.2/10.

Old OP 5: Availability and distribution of, and access to, synthetic DMARDs and biological DMARDs for the treatment of patients with RMDs as well as for patients with COVID-19 (but without RMDs) is a delicate societal responsibility. Therefore, the off-label use of DMARDs in COVID-19 outside the context of clinical trials should be discouraged.

New OP 5: The off-label use of immunomodulatory or immunosuppressive drugs for the treatment of COVID-19 outside of established guidelines, protocols or clinical trials should be discouraged.

The initial fear for a shortage of certain disease-modifying antirheumatic drugs (DMARDs) for patients with RMD (with or without COVID-19), due to overuse for the treatment of patients with COVID-19, which formed an important element of the previous OP 5, has not materialised. As said, hydroxychloroquine is ineffective in COVID-19, and should not be used for that indication anymore. Glucocorticoids (including dexamethasone) are now part of most COVID-19 treatment protocols worldwide and are widely available and shortages are not expected.

After a long period of uncertainty, invoked by RCT with varying results, finally the IL-6R-inhibitor tocilizumab has been proclaimed an effective treatment for COVID-19, in particular for those with severe COVID-19 and largely restricted to the (short) hyperinflammatory phase. The drug has now been included in treatment protocols worldwide, as recommended by the WHO, ${ }^{11}$ which has led to an increased demand for tocilizumab. Still, this increase should be manageable in light of the fact that patients with severe COVID-19 need only one or two intravenous doses and the manufacturer of tocilizumab has had ample time to adapt its production facilities. Therefore, the manufacturer's announcement of global supply constraints of tocilizumab has surprised the professional rheumatological community. EULAR, ACR and the WHO, among others, acted promptly with press-releases, ${ }^{12-14}$ expressing concerns and calling on the company to ensure equitable allocation of current stocks of tocilizumab, and EULAR continues to monitor the availability, distribution and access to this and other medicines. The situation also led to the release of guiding principles by several professional organisations with considerations about the possibility of replacing tocilizumab by compounds with similar mechanism of action, starting new patients on alternative medications or switching intravenous tocilizumab to subcutaneous tocilizumab. ${ }^{1516}$

In view of recent positive delivery developments, this task force decided to suspend the explicit warning about shortage of conventional synthetic DMARDs, but to maintain a general warning against the off-label use of immunomodulatory or immunosuppressive treatments.

Level of agreement: 9.2/10.

\section{GENERAL MEASURES AND PREVENTION OF SARS-COV-2 INFECTION}

The old RCs 1-3 included general public health measures and precautions, meant for patients with RMD without symptoms of SARS-CoV-2 infection, who had not been in contact with SARS-CoV-2 infected patients. By the end of 2020, SARS-CoV-2 vaccination became available and nowadays arguably forms the key measure of prevention of COVID-19 for patients with RMD and beyond.

Old RC 1: Patients with RMDs should be strongly advised to comply with all preventive and control measures prescribed by the health authorities in their countries.

New RC 1: Patients with RMDs should be strongly advised to comply with all infection prevention and control measures 
prescribed by public health authorities, before and after SARSCoV-2 vaccination.

This recommendation remains largely unchanged, but wording is added to reiterate that preventive measures remain important even after (full) vaccination, in order to prevent asymptomatic but infected patients with RMD from unknowingly spreading the virus, even though they may themselves be well protected against severe COVID-19 (hospitalisation, mechanical ventilation and death). Ongoing studies will hopefully reveal to what extent spreading of virus by asymptomatic individuals, as well as mild COVID-19 itself, is prevented by full SARS-CoV-2 vaccination.

Level of agreement: 9.9/10. Level of evidence: 5 .

Old RC 2: Patients with RMDs should in general be advised to comply with the same preventive and control measures as patients without RMDs.

The task force felt that, in analogy with new OP 1 , and by virtue of evolving evidence supportive of new OP 1 , this recommendation had become redundant.

New RC 2: Patients with RMDs should be strongly advised to receive a SARS-CoV-2 vaccination with any of the vaccines approved in their country.

In line with previous EULAR recommendations, issued in December $2020,{ }^{2}$ as well as with evolving evidence outlined in the SLR, ${ }^{4}$ the task force keenly felt that patients with RMDs should be strongly encouraged to receive full SARS-CoV-2 vaccination with one of the approved vaccines. On the basis of the available evidence, the task force was of the opinion that there are no compelling arguments to prioritise or dismiss particular approved vaccines for reasons of less efficacy or increased adverse events, in line with EMA guidance, ${ }^{17}$ even though the two messenger RNA COVID-19 vaccines have been most thoroughly investigated in this regard. However, the task force stipulates that patients and HCP must follow national guidelines that are in place, which may sometimes deviate from EULAR's general principle of equal advisability.

Given that EULAR's remit extends beyond the European Union, and even beyond Europe (as a minority of EULAR countries are not geographically located in Europe), the task force acknowledged that limiting this recommendation to EMAapproved vaccines would not be in the best interest of patients with RMDs living in countries outside the European Union. Therefore, while EULAR encourages vaccine manufacturers to subject not-yet-EMA-approved vaccines to EMA scrutiny and procedures, this recommendation pertains to any vaccine approved in the respective EULAR-affiliated country.

The task force was of the opinion that in the realm of suboptimal SARS-CoV-2 vaccination status worldwide-due to scarcity of vaccines, non-equitable distribution, fear of vaccination or inappropriate vaccination information-it is important to improve vaccination status among the still unvaccinated patients with RMD. Arguably, this is more relevant than administering an additional vaccine dose to those that have already been fully vaccinated and-with exceptions (vide infra) - can be assumed to have a basic level of protection against SARS$\mathrm{CoV}-2$. In line with this position, and in light of the worldwide reach of EULAR recommendations, the task force encourages rheumatology societies of EULAR-affiliated countries to motivate their governments to facilitate the distribution of vaccines from high-income countries to medium-income and low-income countries, so that patients with RMD worldwide can better be protected. The failure of wealthy nations to distribute vaccines to the developing world is likely to result in serious global consequences for the pandemic, promoting the spread and mutation of SARS-CoV-2 among unvaccinated people and the emergence of new and potentially more transmissible and virulent SARSCoV-2 variants.

Level of agreement: 9.6/10. Level of evidence: 3/4.

Old RC 3: Patients with RMDs who do not have suspected or confirmed COVID-19 should be advised to continue their treatment unchanged, namely non-steroidal anti-inflammatory drugs, glucocorticoids, synthetic DMARDs, biological DMARDs, osteoporosis medications and analgesics, among others.

New RC 3: Patients with RMDs who have been vaccinated against SARS-CoV-2 should be advised to continue their treatment unchanged; those who have not been vaccinated should be advised to continue their treatment, taking into account that certain therapies have been associated with an increased risk of severe COVID-19.

The old set of recommendations made a distinction between patients with RMDs (and treatment) at risk of COVID-19 and those who had (already) contracted COVID-19. The somewhat premature advice (old RC 3) to continue drug treatment in patients with symptomless RMD at risk of COVID-19 has proven its validity by evolving evidence, but has also gained dimension by the advent of SARS-CoV-2 vaccines. The new RC 3 makes a distinction between those who have been vaccinated against SARS-CoV-2, and those who have not yet received the vaccine.

The vaccinated patients may, in the opinion of the task force members and based on evolving evidence, safely continue their immunomodulatory or immunosuppressive treatment unchanged, even though an optimal humoral immune response may not occur under treatment. The task force was of the opinion that any protection is better than no protection and that temporarily discontinuing treatment of RMDs bears the risk of flare, and also points to the fact that an optimal immune response against SARS-CoV-2 is not unambiguously defined.

The not (yet) vaccinated patients should realise that the likelihood of severe COVID-19 is increased with certain immunomodulatory or immunosuppressive treatments, as outlined in the SLR,${ }^{4}$ in particular those who are treated with rituximab, MMF, glucocorticoids (discussed under new RC 4) and potentially JAKi. This recommendation should be read as an encouragement to patients and HCP to optimise vaccination status for SARS-CoV-2, taking certain precautions into account (as further outlined below).

Level of agreement: 9.5/10. Level of evidence: 3/4.

\section{MANAGEMENT RMDS WHEN LOCAL MEASURES OF SOCIAL DISTANCING ARE IN EFFECT}

Old RC 4 If the RMD and its drug treatment are stable, and signs or symptoms of drug toxicity are absent, regular blood monitoring and face-to-face rheumatology consultations can be postponed temporarily. If necessary, consultation can take place remotely.

Old RC 5: If the RMD is active, if drug therapy has recently been started or needs adjustment, or if signs or symptoms of drug toxicity emerge, patient and rheumatologist should liaise, weigh the risks of a visit to the clinic against the limitations of remote advice and decide together.

Old RC 6: If a patient with RMD is offered an outpatient, day care or other type of hospital appointment, patients and members of the rheumatology team should follow local guidance for 
infection prevention and control, including the use of personal protection equipment if indicated.

The old recommendations 4-6 advised patients with RMDs on how to act when official restrictions in the freedom of movement apply. They referred to social distancing, varying from keeping 1, 1.5 or 2 metre distance for subpopulations to a complete country-lockdown. When discussing the advisability of these three recommendations, the task force agreed that their content was overtaken by reality and evolving evidence. This does not mean that the recommendations were wrong, or have become obsolete, but rather that the professional rheumatological community and patients with RMD have become accustomed to remote monitoring (old RC 4), initiating DMARD treatment during the pandemic (old RC 5) and triaging those who need a face-to-face consultation (old RC 6). Therefore, the task force decided to remove these three previous recommendations and further refer for this matter to EULAR guidance about remote monitoring in development.

\section{MANAGEMENT OF COVID-19 IN THE CONTEXT OF RMDS}

Old recommendations $7-10$ referred to scenarios in which a patient with RMD had been in contact with a SARS-CoV-2 infected patient or had become infected themselves, with a focus on the use of immunomodulatory or immunosuppressive drugs.

Old RC 7: Patients with RMDs without COVID-19 symptoms who have been in contact with a SARS-CoV-2-positive person should be tested for SARS-CoV-2 themselves.

While in April 2020 this recommendation still raised dissent among task force members, due to the scarcity of SARS-CoV-2 tests and uncertainty about the potential consequences of a positive test result (eg, the need to pause drugs), this was no longer a source of discussion anymore in July 2021. SARS-CoV-2 testing has become ubiquitous and part of usual clinical care. The old RC 7 was considered redundant by the task force and therefore removed.

Old RC 8: If a patient with RMD and symptoms of COVID-19 is chronically treated with glucocorticoids, this treatment should be continued.

New RC 4: If a patient with RMD receiving long-term glucocorticoid treatment develops suspected or confirmed COVID-19, this treatment should be continued.

In spite of several studies pointing to an association between glucocorticoid use and worse COVID-19 prognosis, extensively outlined in the SLR, ${ }^{4}$ old RC 8 (renumbered as new RC 4) has stood the test of time. After studying the results of the SLR, the task force came to the conclusion that the observed association between glucocorticoid-exposure and severe COVID-19 could well be explained by confounding by indication, with the confounder being disease activity, which has also been associated with a worse COVID-19 prognosis. ${ }^{78}$ The suggestion of a glucocorticoid dose response that was seen in a few studies may reinforce this conclusion. While an adverse effect of glucocorticoids themselves cannot be entirely excluded, there is also sparse indirect evidence in the literature that pausing or discontinuing glucocorticoids for reasons of safety is associated with disease flaring, which in itself may contribute to an adverse outcome of COVID-19. Finally, it should also be noted that patients on longterm glucocorticoid therapy are at risk of glucocorticoid-induced adrenal suppression and may therefore require glucocorticoid supplementation in the context of major trauma, surgery or significant intercurrent infection, including COVID-19. ${ }^{18}$
The advice to continue glucocorticoids in patients with RMD without symptoms of COVID-19 is now covered by the generic new RC 3; the advice to continue glucocorticoids in patients with RMD with suspected or proven COVID-19 is covered by new RC 4. The task force remains of the opinion that the principle of 'lowest possible dose' as per existing EULAR-recommendations for the management of medium-dose to high-dose glucocorticoids therapy is part of good clinical practice and valid under all circumstances. ${ }^{19}$

Level of agreement: 9.3/10. Level of evidence: 3/4.

New RC 5: If a patient with RMD receiving rituximab treatment contracts SARS-CoV-2, postponing the next cycle of rituximab should be considered

This new recommendation without precedent in the first set was included because of evolving evidence that patients who use B-cell depleting therapy (in particular anti-CD20 therapy with rituximab) for their RMD have a higher risk of developing severe COVID-19 and an inferior antibody response to SARS-CoV-2 vaccination. ${ }^{4}$ The task force realised that there are many practical questions around the best possible management of patients with RMD treated with B-cell-depleting therapy. Other professional organisations than EULAR have sometimes provided more granular recommendations about B-cell-depleting therapy in association with COVID-19. ${ }^{20}$ This task force was of the opinion that an evidence-based recommendation on how to act in specific circumstances is not opportune, since the data proving that specific measures are indeed effective and safe are currently lacking. Still, the task force felt some pressure of sister organisations to make recommendations regarding rituximab, administered in cycles with intervals ranging from 1 to 12 months. This recommendation, as well as the ones pertaining to vaccination that follow below, is based on expected effects of rituximab and clinical feasibility, rather than on solid evidence. In general, for patients on rituximab, the task force found it reasonable to postpone a next cycle of rituximab (or, alternatively, to replace rituximab by an equally effective drug) in a patient with stable RMD as long as the clinical situation allows a delay. While the task force recognises some excess risk of rituximab in such circumstances, a contraindication for rituximab is relative, not absolute.

Level of agreement: 9.7/10. Level of evidence: $3 / 4$.

Old RC 9: If patients with RMDs experience mild symptoms of COVID-19, potential treatment changes in DMARDs should be discussed on a case-by-case basis.

This old recommendation reflected a compromise between task force members who considered the continuation of DMARDs in a patient with RMD with symptoms of COVID-19 undesirable, and those who agreed with the argument that more than $90 \%$ of patients with COVID-19 usually experience a mild and self-limiting course, and that early data did not point to a significantly increased risk of severe COVID-19 in patients with RMD on DMARD treatment. Since then, the ever-increasing body of evidence has tipped the balance towards a more moderate and lenient attitude of continuing DMARDs in case of mild COVID-19 symptoms. Herewith, this old RC 9 has become redundant, and its content is now entirely covered by new RC 3 .

Old RC 10: Patients with RMDs and initially mild symptoms who experience worsening of COVID-19 symptoms should immediately seek further healthcare advice of an expert in treating COVID-19, such as a pulmonologist, an internist or a specialist in infectious diseases, dependent on local circumstances. 
New RC 6: Patients with RMDs and initially mild symptoms who experience worsening of COVID-19 symptoms should immediately seek further healthcare advice from an expert in treating COVID-19.

While consensus has now been obtained regarding the continuation of DMARDs in a patient with mild COVID-19, it is still opportune to advise on patients with RMD with worsening of COVID-19. They should be referred to an expert in treating COVID-19, not being the rheumatologist, as per new RC 6 .

It has become clear during the pandemic that a small minority of patients with COVID-19 will experience a more severe course. Patients with severe COVID-19, with or without RMDs, may require ventilatory support, antibiotic treatment, anticoagulation and temporary immunomodulatory or immunosuppressive treatment. While some of these treatments involve medications with which rheumatologists are considered broadly familiar, the task force is (still) of the opinion that the diagnosis of severe COVID-19, the indication to start adjunctive therapy and the monitoring of the course of severe COVID-19 belong to the realm of an expert in COVID-19 (new OP 2). This does not mean that rheumatologists should not be involved in the design of-and discussion about-protocols and guidelines, as per new OP 4. For more details about the immunomodulatory treatment of (severe) COVID-19 per se, the task force refers to the EULAR's points to consider on the use of immunomodulatory therapies in COVID-19. ${ }^{21-24}$

Level of agreement: 9.9/10. Level of evidence: 3/4.

Old RC 11: Patients with RMDs who are admitted to the hospital because of significant COVID-19 should follow local treatment recommendations for COVID-19 as applied by the treating expert.

This recommendation dates back to the time at which task force members made a deliberate distinction between patients with mild COVID-19, those with worsening of once mild COVID-19 and those with significant or severe COVID-19. This distinction has gradually become outdated and redundant for the advice of how to manage patients with RMD with symptoms of COVID-19 today. Those with mild symptoms may continue their treatment unchanged and followed up until recovery, as per new RC 3. Those with worsening symptoms should be referred to an expert in COVID-19 without exception, as outlined in new RC 6. Herewith, old RC 11 has become redundant.

\section{PREVENTION OF OTHER INFECTIONS THAN SARS-COV-2}

Old recommendations 12 and 13 intended to remind the rheumatologist of potentially coexisting comorbid infections for which regular vaccinations exist (old RC 12), and of other important infectious diseases that could phenotypically mimic COVID-19 (old RC 13).

Old RC 12: Patients with RMDs without symptoms of COVID-19 should be advised to update their vaccination status in accordance with the EULAR-recommendations for the vaccination of patients with RMDs, with a particular focus on pneumococci and influenza.

New RC 7: Patients with RMDs should be advised to update their general vaccination status in accordance with the EULARrecommendations for the vaccination of patients with RMDs, with a particular focus on pneumococci and influenza.

This recommendation was essentially unchanged. The update of EULAR recommendations for vaccination in adult patients with autoimmune inflammatory rheumatic diseases was published in 2019 and should be consulted for further information. ${ }^{25}$

Level of agreement: 9.7/10. Level of evidence: 5 .

Old RC 13: In patients with RMDs treated with cyclophosphamide or glucocorticoids, pneumocystis Jiroveci pneumoniaprophylaxis should be considered

This recommendation pertaining only to a small minority of patients with RMDs, particularly those with intensive immunosuppressive therapy, served to alert the rheumatologist's attention to a phenotypical mimic of COVID-19 at a time at when confirmatory COVID-19 testing was not self-evident. The task force was of the opinion that clinical confusion between Pneumocystis jiroveci pneumonia (PJP) and COVID-19-pneumonia has become unlikely. While PJP-prophylaxis remains highly topical for those at risk of PJP due to (severe) immunosuppression, the task force was of the opinion that this is out of the scope of the current manuscript and the old RC 13 could be deleted.

\section{NEW RECOMMENDATIONS}

The task force added two recommendations referring to SARSCoV-2 vaccination that had no precedent in the old set of recommendations.

New RC 8: In patients with RMDs not using immunomodulatory or immunosuppressive treatment, SARS-CoV-2 vaccination should precede a treatment start with such therapy if clinically feasible.

This recommendation finds its justification in recent evidence, summarised in the SLR, ${ }^{4}$ pointing to an impaired humoral immune response in patients with RMD treated with particular immunomodulatory or immunosuppressive treatments. The level of impairment varies by compound: from the suppression of humoral immune response in case of B-cell-depleting therapy and MMF, to the generally mild-to-moderate impairment in case of methotrexate, glucocorticoids and JAKi, to no distinguishable impairment for tumour necrosis factor (TNF)inhibitors and IL-17-inhibitors, as well as for most conventional synthetic DMARDs. While the task force agreed that the clinical significance of an impaired level of antibodies to SARS-CoV-2 (humoral immune response) is still unclear, it also argued that it makes sense to first vaccinate and then start with immunomodulatory or immunosuppressive therapy, unless the delay of treatment is damaging or life threatening, a consideration that is left at the discretion of the rheumatologist and the patient in shared decision-making.

Level of agreement: 9.6/10. Level of evidence: 3/4.

New RC 9: In patients with RMDs using rituximab or another B-cell depleting therapy, SARS-CoV-2 vaccination should be scheduled in a way to optimise vaccine immunogenicity.

This new recommendation serves to bring the EULAR recommendations in sync with guideline documents of professional sister organisations that have recommended explicitly on this matter. ${ }^{26}$ It draws the rheumatologist's, HCP's and patient's attention to the fact that-as outlined above several times-Bcell depleting therapy may compromise the development of an appropriate (humoral) defence against SARS-CoV-2 on vaccination. While new RC 8 points to postponement of the start of immunomodulatory or immunosuppressive treatment when clinically feasible, it does not suffice for patients who have already been treated with cycles of rituximab, which may surely cause a long-lasting and not immediately reversible functional 
suppression of B-cell activity. The task force acknowledged that patients and HCP may ask for more specific guidance in terms of a minimal duration between the last cycle of rituximab and the vaccination, but had to conclude that such a time frame does not logically follow from the currently available data; the highly variable B-cell repopulation kinetics may in fact be a more important factor to take into account when deciding when to vaccinate rather than a specific timeframe. The task force acknowledges that the advice to optimize vaccine immunogenicity [sic] without further explanation may not fully cover patients' and HCP's expectations. However, in the absence of evidence, although in spite of existing guidance from other organisations, the task force feels they could not be more specific at this point in time.

Level of agreement: 9.6/10. Level of evidence: $3 / 4$.

\section{NEW POINTS TO CONSIDER}

At the same time as real-world effectiveness data of SARS-CoV-2 vaccination emerged over the last few months, pre-emptive action was undertaken by governments of many countries, fuelled by public opinion and anxiety among health experts regarding waning vaccine effectiveness. These countries implemented two types of strategies, namely:

1. Administering an additional dose of the vaccine to individuals who had received their primary course of vaccination while on immunomodulatory or immunosuppressive drugs, or to individuals with an underlying health condition causing a primary or acquired immunodeficiency state (third primary dose (or: second primary dose if the initial vaccine administered was a single-dose vaccine) - for simplicity this manuscript will consistently refer to the term third primary dose); and:

2. Reinforcing immunisation of the vaccinated population with a booster vaccine dose, usually starting with priority groups (such as: older and more vulnerable individuals) and potentially expanding this strategy to the entire vaccinated population.

Based on these developments, EULAR updated its SLR with the most recent evidence on vaccination of patients with RMDs, and re-opened the discussion about the desirability of recommendations pertaining to revaccination of previously vaccinated patients with RMD.

Two additional statements emerged from this discussion, informed by the post hoc data of the SLR.

It was decided that these statements did not deserve a status of recommendation, since supportive evidence was fragmentary, often unconfirmed and methodologically not robust enough. However, the task force was also of the opinion that EULAR cannot ignore public health advice by authorities at the countries' level, and that patients with RMDs and the HCPs taking care of them value the opinion and guidance of EULAR. Therefore, the task force chose the formulation of points to consider (PtC), in order to convey the message of immaturity of the evidence regarding revaccination, on the one hand, and the appreciation of-and compliance to-precautionary public health measures issued by authorities under conditions of uncertainty, on the other hand. The two points to consider are read as follows:

New PtC 1: There are concerns that individuals on certain immunosuppressive or immunomodulatory drugs may not mount an adequate protective response to COVID-19 vaccination. Data are not currently available to reliably identify who might, or might not, benefit from a third primary dose of a SARS-CoV-2 vaccine. Taking a precautionary position, third primary doses are being recommended by some authorities in selected groups of individuals and EULAR supports this approach.

Here the task force elaborated on evidence that some patients with RMD may not mount a full immune response to COVID-19 vaccination. This has been well-documented for patients exposed to anti-CD20-therapy during vaccination, who have been shown to have impaired (or even absent) humoral response to the vaccine, and on accruing evidence that this latter might convey an increased risk of (severe) COVID-19, as per RCs 8 and 9. However, many uncertainties remain. First, impaired humoral immunity is not the same as no protection, and for example studies looking at cellular responses have largely been reassuring, even in the absence of a humoral response (there is no proper correlate of protection). Second, proving an association between the level of humoral immunity and the risk of (severe) COVID-19 neither means that re-vaccination will improve humoral immunity in these patients, nor that improving humoral immunity by re-vaccination will reduce the risk of (severe) COVID-19. Epidemiological studies that allow such a causal chain of argumentation are lacking so far and the potential for additional protection from a third primary dose is unknown at an individual level.

As argued before, however, authorities in several (highincome) countries have already issued guidance that immunosuppressed patients (among which patients with RMD who use certain immunomodulatory or immunosuppressive drugs) should receive a third primary dose. This approach is based on the assumption that a third primary dose is unlikely to confer significant harms or disadvantages, but may offer the possibility of benefit. So far, there is no unanimity about which patients and which drugs are critical in this regard. Following the information from the SLR, the task force is of the opinion that the data on anti-CD20 therapy are most compelling, followed by data on MMF and glucocorticoids (potentially at higher dosages, but a dose-dependent effect and potential dosage cut-off are still unclear). Data on methotrexate, JAKi and abatacept are not (yet) consistent/robust. Reassuringly, the use of hydroxychloroquine and some targeted therapies (eg, TNF-inhibitors, IL-17inhibitors, IL-6R blockers, belimumab) have not been associated with lower antibody responses. Data are scarce (or lacking at all) for other conventional synthetic/targeted synthetic DMARDs (eg, sulfasalazine, leflunomide, apremilast), other biological DMARDs (eg, IL-12/23-inhibitors, IL-1-inhibitors) and other immunosuppressive drugs (eg, cyclophosphamide, cyclosporine, azathioprine and tacrolimus).

The task force recognises the positions taken by authorities in several countries, usually fueled by expert committees in those countries and largely based on expert-opinion or expertsuspicion, even though different authorities may delineate different groups of individuals to whom the guidance should refer. The task force stipulates that support of a third primary dose vaccination policy does not mean that EULAR recommends this approach on the basis of firm scientific evidence at this point in time. The decision to administer third primary doses (or not) is the outcome of shared decision-making by the physician and the well-informed patient.

Level of agreement: 9.7/10. Level of evidence: 5 .

New PtC 2: There are concerns that protection provided by vaccines against severe COVID-19 decreases gradually over time. Insufficient time has passed to know what levels of protection might be expected beyond 4-6 months after the primary course. Taking a precautionary position, booster doses are being recommended by several authorities and EULAR supports this approach. 
Table 2 Research agenda

General measures and prevention of SARS-CoV-2 infection

1. Large unselected registry studies to assess the course of COVID-19 in patients with rare autoimmune diseases compared with the general population.

Management of immunosuppressive or immunomodulatory drugs in patients with RMD with COVID-19

1. Large unselected registry studies to assess the risk of Janus kinase inhibitors and immunosuppressants (glucocorticoids, azathioprine, cyclosporine, cyclophosphamide, mycophenolate and tacrolimus) on a worse course of COVID-19.

2. Studies to assess the impact of other B-cell depleting strategies (eg, belimumab) on the outcome of SARS-CoV-2 infection and COVID-19 diseases course.

3. Studies to compare different disease-modifying antirheumatic drugs management strategies in the context of SARS-CoV-2 infection: unchanged, versus dose reduction versus interruption in patients with RMDs.

Vaccination of the patients with RMD

1. Studies to assess the impact of temporarily stopping medications 'of concern' before or after SARS-CoV-2 vaccination and supplemental (booster) dosing, in order to improve immunogenicity, and the impact of such strategies on disease activity and need of additional treatments, for example, glucocorticoids.

2. Studies to assess the impact of an additional dose as part of the initial primary SARS-CoV-2 vaccination in selected subsets of patients with RMDs, in order to improve the humoral and/or cell-mediated immunity to SARSCoV-2 vaccines.

COVID-19, coronavirus disease 2019; RMDs, rheumatic and musculoskeletal diseases; SARS-CoV-2, severe acute respiratory syndrome coronavirus 2.

Here, the task force took the better safe than sorry approach. The argument of waning protection is based on waning humoral immunity against SARS-CoV-2 over time, which is an 'intermediate outcome'. Robust epidemiological evidence that waning immunity is a particular problem in patients with RMD (and if yes, how long after completion of a primary vaccine series?) is lacking so far, but this is conceivable from a theoretical perspective. Some evidence that a booster vaccine in such circumstances improves protection in the general population now also starts to accrue. ${ }^{2728}$ Following guidance issued by an increasing number of governments, especially in high-income countries, and knowing that booster vaccination is likely a relatively safe medical intervention, also in patients with RMD, the task force decided to take a passive but supportive stand. It means that the task force has understanding of the authorities' approach (and its potential benefit in fighting the pandemic), rather than that the task force is of the opinion that booster vaccination has undeniably proven its merits (yet).

Level of agreement: 9.4. Level of evidence: 4.

\section{DISCUSSION}

This set of five overarching principles, nine recommendations and two points to consider forms the first update of the original EULAR provisional recommendations for the management of patients with RMDs during the SARS-CoV-2 pandemic. The scientific status of the first set was meagre, but the level of evidence of the updated recommendations has significantly improved, in accordance with evolving knowledge. However, there are still many unknowns. Despite an exponential and likely unprecedented explosion of scientific studies, many critical clinical questions, some of which are mentioned in the research agenda (table 2), have not yet been fully addressed in clinical studies and remain largely unanswered. While the overall impression is not a negative one, the majority of available studies still received the predicate of unclear or high risk of bias. Those few studies with low risk of bias, the best ones so to say, have had a significant impact on the reformulation of the old recommendations into new ones.

\section{Old versus new recommendations}

When comparing the old and new recommendations, a few observations stand out.

The first is that the number of recommendations has reduced from 13 to 9 and the length of each recommendation has importantly reduced too. This may seem a trivial observation without scientific meaning, but may also testify to an increased maturity of the field and (consequently) more unanimity among task force members. That levels of agreement were (even) higher than in the previous set, adds to the credibility of the latter. In April 2020 diverging opinions, due to lack of available evidence and a necessary reliance on (sparse) experience (not to say: beliefs), had materialised into a rather high number of rather verbose recommendations, in order to better reflect different, sometimes even opposing opinions. In July 2021, after properly being informed by the SLR-committee, the task force reached consensus within 3 hours of discussion, and delivered nine concise and structured recommendations.

The second observation is that the content of the updated set is dominated by SARS-CoV-2 vaccination. SARS-CoV-2 vaccination is indisputably an example of unprecedented medical progress. While in April 2020 the prospect of SARS-CoV-2 vaccination was still uncertain, in July 2021 a significant proportion of the population in many EULAR countries were already vaccinated and discussions about (and implementation of) a third primary dose had started, although confirmatory evidence for that policy is still lacking.

More focus on vaccination also illustrates the progress that has been made in understanding the hazards that patients with RMD face in the context of COVID-19. Because the risk of poor outcomes in general is increased in several RMDs, many had feared that patients with RMDs were not only at higher risk of contracting COVID-19, but would also experience a worse course when having COVID-19. In spite of a couple of exceptions and uncertainties, amply described in the accompanying SLR, ${ }^{4}$ and some disagreement among task force members, this fear has not become manifest and the updated set of recommendations is a good reflection of that appreciation; patients with RMD are not very different from unaffected individuals in the general population (even though they may have a higher comorbidity burden), most treatments can be safely continued and special precautions for patients with RMD (beyond those advised for the general population) are in general not necessary.

This does not mean that there are no outstanding questions. JAKi and (even) sulfasalazine have recently been associated with an increased risk of severe COVID-19, rituximab is a notoriously difficult therapy to manage in the context of COVID-19 and vaccination and there are also question marks about some truly immunosuppressive drugs such as MMF, a drug prescribed for several systemic autoimmune diseases, about which the first impressions were slightly worrisome. Still, the make-up of the studies that released these associations preclude a causal interpretation; selection bias and confounding-by-indication, rather than the drug itself, may be responsible for the reported excess risk in many studies. 


\section{New EULAR recommendations in context}

Comparing these EULAR recommendations with other recent recommendations, such as the latest version of the ACR recommendations, ${ }^{26}{ }^{29}$ reveals, as expected, high levels of similarity. Issues of controversy are of relatively minor importance. The ACR has released guidance documents that have been more frequently updated than EULAR's, and are far more detailed, since they deal with several scenarios and drugs separately. ${ }^{2629} \mathrm{~A}$ main discrepancy pertains to ACR's recommendation of a drugpause for most DMARDs in case of known or suspected SARSCoV-2 exposure. ACR also advises to pause DMARDs in case of active or presumptive COVID-19 (exceptions are sulfasalazine and, conditionally, IL-6 inhibitors). Reinitiating treatment should, according to the ACR, depend on COVID-19 symptom resolution (after at least 7-14days, or more for certain DMARDs). The British Society of Rheumatology ${ }^{16}$ and the UK's National Institute of Clinical Excellence ${ }^{30}$ also advise to pause DMARDs for a while in case of manifest COVID-19. The EULAR task force is more lenient in this regard, since it does neither recommend to pause in case of exposure to SARSCoV-2, nor in case of mild symptomatic COVID-19 (ie, the large majority of patients with COVID-19 that do not require oxygen supplementation or hospitalisation). In case of more severe (eg, hospitalised) COVID-19, EULAR leaves the decision about pausing or stopping DMARDs at the discretion of the treating physician for COVID-19 (new OP 2), in consultation with the treating rheumatologist (new OP 3). Whether this discrepancy in policies results from a different interpretation of the available literature, from different local circumstances or from differences in medicolegal context between Europe and the USA, is unclear.

Regarding SARS-CoV-2 vaccination, EULAR has aggregated management recommendations and vaccination recommendations into one document. The ACR has recently released a guidance document entirely dedicated to SARS-CoV-2 vaccination in patients with RMDs. ${ }^{31}$ The ACR has provided no less than 76 guidance statements to cover all possible scenarios that patients with RMDs may encounter. Basically, these ACR-statements are in line with EULAR's simple and concise recommendation that all patients with RMDs, without exception, should be fully vaccinated as soon as possible (new RC 2). The ACR provides more detailed guidance on how to manage patients with RMD in specific scenarios (the ACR, for instance, advises to pause certain DMARDs around vaccination, gives specific advice per DMARD and provides timelines). The EULAR task force was aware of the ACR document, and discussed these matters, but was essentially of the opinion that the available scientific evidence precluded such a detailed level of advice. The task force decided that a more generic advice was opportune (new RC 2), which could rely on a very high level of agreement among task force members.

\section{Addition of points-to-consider into context}

Mixing recommendations and points-to-consider in one EULAR manuscript is unprecedented, but likely justifiable in the context of the unprecedented pandemic with rapidly evolving evidence and changing scenarios. What remains to be discussed is the realisation that public health advice (including medical interventions) by governments in different countries is not always driven by solid scientific evidence, but also by public (and experts') beliefs and perceptions, and by emotions. The EULAR task force struggled with this issue, for which current SOPs do not provide resolution. The task force finally compromised that EULAR will not publicly contest official guidance issued by individual EULAR member states, but will report their conclusions based on their interpretation of the evidence and according to the rules laid down in their SOP.

The final outcome, in clinical practice, is always the result of the process of shared decision-making between the patient, who is optimally informed about facts and residual uncertainties, and the HCP.

\section{A critical appraisal of evolving epidemiological evidence on SARS-CoV-2/COVID-19}

Translating scientific evidence, stemming from high-profile epidemiological surveys, RCTs or high-quality observational studies, to the situation of the individual patient in daily clinical practice is not an easy task. Communicating such information accurately to patients is even more difficult. Big-data studies and multicountry epidemiological registries will often attract most attention from physicians and lay press, because of the high numbers of patients involved in such studies. Not infrequently do these studies report small but statistically significant excess risks for patients with RMD in comparison to the general population. It is of utmost importance for HCP, who have to deal with individual patients rather than an entire population of patients, to realise that a small excess risk (risk estimates arbitrarily between 1 and 2-3) is often irrelevant if the base case risk for that patient is low (eg, less than 1 in 100), even if the small excess risk is highly statistically significant. The anticipated consequence (eg, lower risk of severe COVID-19) of a certain interventional recommendation (eg, DMARD pausing), seemingly justified by an excess risk at the group level, should always be weighed against unwarranted and often unforeseen consequences of that interventional recommendation (eg, relapse of disease activity). In addition, the task force realised that the technical demonstration of an observational association between an exposure (eg, the use of a DMARD) and an outcome (eg, hospitalisation for COVID-19) alone does not constitute sufficient evidence to recommend an intervention (eg, pausing the DMARD, revaccination) if the proof that such an intervention really works is lacking.

On the other hand, the task force sometimes expressed support for proposed interventions with potential but largely theoretical benefit and little harm to expect (a good example is revaccination of patients with RMD), but reiterated in such cases that the scientific evidence was lacking.

\section{CONCLUSION}

The task force hopes that these updated, now more evidencebased, recommendations on how to manage patients with RMDs in the context of SARS-CoV-2 and COVID-19 give HCPs the tools to make clinical decisions about SARS-CoV-2 prevention, DMARD management and SARS-CoV-2 (re)vaccination. More importantly, it hopes that it will help build confidence among patients with RMDs that, (in general), their risk of severe COVID-19 is not importantly increased and that SARS-CoV-2 (re)vaccination, crucial to finally contain the pandemic, can safely take place.

\footnotetext{
Author affiliations

${ }^{1}$ Clinical Immunology \& Rheumatology, Amsterdam UMC Locatie AMC, Amsterdam, North Holland, The Netherlands

${ }^{2}$ Department of Rheumatology and Clinical Immunology, Zuyderland Medical Center, Heerlen, The Netherlands

${ }^{3}$ Rheumatology, Leiden University Medical Center, Leiden, The Netherlands ${ }^{4}$ Rheumatology, Zuyderland Medical Centre Heerlen, Heerlen, The Netherlands ${ }^{5}$ Life, Health and Environmental sciences, internal medicine and nephrology unit, University of I'Aquila, I'Aquila, Italy
} 
${ }^{6}$ Institute of Infection Immunity and Inflammation, University of Glasgow College of Medical Veterinary and Life Sciences, Glasgow, UK

${ }^{7}$ Rheumatology, University Medical Center Utrecht Department of Rheumatology and Clinical Immunology, Utrecht, The Netherlands

${ }^{8}$ Rheumatology and Clinical Immunology, Charité University Hospital, Berlin, Germany

${ }^{9}$ Department of Clinical Sciences and Community Health, ASS G. Pini, University of Milan, Milano, Lombardia, Italy

${ }^{10}$ Immunorhumatologie, CHU Lapeyronie, Montpellier, France

${ }^{11}$ Rheumatology, Saint James's Hospital, Dublin, Ireland

${ }^{12}$ University of Alabama at Birmingham Department of Medicine, Birmingham, Alabama, USA

${ }^{13}$ Rheumatology, Tel Aviv Medical Center, Tel Aviv, Israel

${ }^{14}$ INSERM, Institut Pierre Louis d'Epidémiologie et de Santé Publique, INSERM, Sorbonne Universite, Paris, France

${ }^{15}$ APHP, Rheumatology department, Hopital Universitaire Pitie Salpetriere, Paris, France

${ }^{16}$ Rheumatology \&Clinical Immunology, University Medical Center Utrecht, Utrecht, The Netherlands

${ }^{17}$ 2nd Department of Medicine, Hietzing Hospital Vienna, Vienna, Austria

${ }^{18}$ Scienze Cliniche e Biologiche, Università degli Studi di Torino, Torino, Italy

${ }^{19}$ Institute of Translational and Clinical Research, Newcastle University and Musculoskeletal Unit, Newcastle upon Tyne Hospitals NHS Foundation Trust, Newcastle upon Tyne, UK

${ }^{20}$ Centre of Rheumatology, Buda Hospital of the Hospitaller Order of Saint John of God, Budapest, Hungary

${ }^{21}$ Cyprus League for People with Rheumatism, Limassol, Cyprus

${ }^{22}$ Rheumatology, Assistance Publique-Hôpitaux de Paris (AP-HP), Hôpitaux

universitaires Paris-Sud - Hôpital Bicêtre, Le Kremlin Bicêtre, France

${ }^{23} 3$ Université Paris-Sud, Center for Immunology of Viral Infections and Auto-immune

Diseases (IMVA), Institut pour la Santé et la Recherche Médicale (INSERM) UMR

1184, Université Paris-Saclay, Le Kremlin Bicêtre, France

${ }^{24}$ MVLS College Office, University of Glasgow, Glasgow, UK

${ }^{25}$ Institute of Infection, Immunity and Inflammation, University of Glasgow, Glasgow, UK

${ }^{26}$ Centre for Inflammation and Tissue Repair, UCL Respiratory Division of Medicine, University College London Hospitals NHS Foundation Trust, London, UK

${ }^{27}$ Rheumatology, University College London Hospitals NHS Foundation Trust, London, UK

${ }^{28}$ Rheumatology and Clinical Immunology, Giessen University, Bad Nauheim, Germany

${ }^{29}$ Division of Rheumatology and Clinical Immunology, Internal Medicine IV, LudwigMaximilians-Universitat Munchen, Munchen, Germany

${ }^{30}$ Medicine 3, division of Rheumatology, Medical University of Vienna, Wien, Austria

${ }^{31}$ Deutsche Rheuma-Liga, Bonn, Germany

${ }^{32}$ Schools of Medicine and Public Health, division of Infectious Diseases, Oregon Health \& Science University, Portland, Oregon, USA

${ }^{33}$ Rheumatology, La Paz University Hospital, Madrid, Spain

${ }^{34}$ Centre for Rheumatology and Department of Neuromuscular Diseases, University College London Hospitals (UCLH) NHS Foundation Trust, London, UK

${ }^{35}$ Biomedical Research Centre, University College London Hospitals NHS Foundation Trust, London, UK

${ }^{36}$ Rheumatology, Northwick Park Hospital, London North West University Healthcare NHS Trust, London, UK

Twitter John D Isaacs@ProfJohnlsaacs, Puja Mehta@DrPujaMehta1 and Pedro M Machado @pedrommcmachado

Contributors All authors contributed to and finally approved the current manuscript.

Funding JDI is a National Institute for Health Research (NIHR) senior investigator and work in his laboratory is supported by the Research into Inflammatory Arthritis Centre Versus Arthritis and the NIHR Newcastle Biomedical Research Centre in Ageing and Long-term Conditions. PMM is supported by the NIHR University College London Hospitals (UCLH) Biomedical Research Centre (BRC). The views expressed are those of the authors and not necessarily those of the (UK) National Health Service (NHS), the NIHR or the (UK) Department of Health and Social Care.

Competing interests RBML received honoraria for lecturing and consultation from AbbVie, Amgen, BMS, Celgene, Galapagos, Gilead, Janssen, Eli Lilly, Novartis, Pfizer, UCB, is chair of EULAR's committee for the quality of care and is owner and director of Rheumatology Consultancy BV. RBML is chair of EULAR's committee of quality of care. AN received honoraria for lectures and consulting form BMS, UCB, Chigai and Roche. JWJB received honoraria for lectures and consulting from Fresnius, Galapagos, Syneos. GRB received honoraria for lectures and consulting from AbbVie, Amgen, BMS, Gilead, Janssen, Lilly, Novartis, Pfizer, Sanofi-Aventis, Roche, UCB. RC received honoraria for lectures from $A b b V i e$, Janssen, Roche, Sanofi. BC received honoraria for lecturing and consultation from AbbVie, BMS, Celltrion, Galapagos, Gilead, Janssen, Eli Lilly, Merck, Novartis, Pfizer, Roche-Chugai. RC received honoraria for lectures and consulting or travel support from AbbVie, Janssen, Nordic Pharma, Roche and Sanofi. JRC received research grants and consulting from Amgen, AbbVie, BMS, GSK, Janssen, Lilly, Novartis, Pfizer, and chairs the ACR COVID Vaccine Guidance Task Force. OE received honoraria for lecturing and consultation from AbbVie, BMS, Celgene, Gilead, Janssen, Eli Lilly, Novartis, Pfizer and B.I. MWH Consultation and speaker's fees from ALK and Roche. LH received a speaker's fee from Grünenthal, honoraria for writing articles from MedMedia, and travel support from AbbVie, Biogen, BMS, MSD, Novartis and Roche. Al received consultancies, honoraria, speaker-fees from AbbVie, MSD, Alfasigma, Celltrion, BMS, Celgene, Eli Lilly, Sanofi, Genzyme, Pfizer, Galapagos, Gilead, Novartis, SOBI and research grants from MSD, Alfasigma, AbbVie. Al is acting president of EULAR. JDI received research grants from Pfizer, Janssen and GSK and honoraria for lectures, conference support from Eli Lilly and Gilead, and speaker/consulting fees from AbbVie, Gilead, Roche and UCB. XM received consulting fees from BMS, Gilead, Janssen, Pfizer, Samsung, UCB. $B C$ received honoraria from AbbVie, BMS, Gilead, Janssen, Lilly, Merck, Novartis, Pfizer, Roche-Chugai, Sanofi and UCB; and research grants from Novartis, Pfizer, and Roche. IBM received research grants from Lilly, Pfizer, BMS, Celgene, Janssen; and consulting fees from AbbVie, BMS, Celgene, Gilead, Janssen, Lilly, Novartis, Pfizer, Sanofi-Aventis and UCB. IBM is past president of EULAR. HS-K received honoraria for lectures and consulting from AbbVie, Amgen, BMS, Gilead, Janssen, Lilly, Novartis, Pfizer, Sanofi-Aventis, Roche and UCB. JSS received grants to his institution from AbbVie, AstraZeneca, Janssen, Lilly, MSD, Pfizer and Roche and provided expert advice for, or had symposia speaking engagements with, AbbVie, Amgen, AstraZeneca, Astro, Bristol Myers Squibb, Celgene, Celltrion, Chugai, Gilead, ILTOO Pharma, Janssen, Lilly, MSD, Novartis-Sandoz, Pfizer, Roche, Samsung, Sanofi and UCB. JSS is editor of the Annals of Rheumatic Diseases. KW received honoraria and consultancy fees from AbbVie, Lilly, Roche, GSK, Novartis, BMS, Pfizer, UCB, Janssen, Regeneron and Sanofi. VN-C received research grants/honoraria from AbbVie, Janssen, Lilly, Novartis, Pfizer, and UCB. PMM received consulting/speaker's fees from AbbVie, BMS, Celgene, Eli Lilly, Galapagos, Janssen, MSD, Novartis, Pfizer, Roche and $U C B$, all unrelated to this manuscript.

Patient and public involvement Patients and/or the public were involved in the design, or conduct, or reporting, or dissemination plans of this research. Refer to the Methods section for further details.

Patient consent for publication Not applicable.

Ethics approval This study does not involve human participants.

Provenance and peer review Not commissioned; externally peer reviewed.

\section{ORCID iDs}

Robert B M Landewé http://orcid.org/0000-0002-0577-6620

Féline P B Kroon http://orcid.org/0000-0002-8940-0582

Alessia Alunno http://orcid.org/0000-0003-1105-5640

Aurélie Najm http://orcid.org/0000-0002-6008-503X

Johannes WJ Bijlsma http://orcid.org/0000-0002-0128-8451

Gerd-Rüdiger R Burmester http://orcid.org/0000-0001-7518-1131

Richard Conway http://orcid.org/0000-0003-2538-3362

Laure Gossec http://orcid.org/0000-0002-4528-310X

Annamaria lagnocco http://orcid.org/0000-0001-5592-724X

John D Isaacs http://orcid.org/0000-0002-6103-7056

Xavier Mariette http://orcid.org/0000-0002-4244-5417

Puja Mehta http://orcid.org/0000-0001-9459-9306

Hendrik Schulze-Koops http://orcid.org/0000-0002-1681-491X

Kevin L Winthrop http://orcid.org/0000-0002-3892-6947

Victoria Navarro-Compán http://orcid.org/0000-0002-4527-852X

Pedro M Machado http://orcid.org/0000-0002-8411-7972

\section{REFERENCES}

1 Landewé RB, Machado PM, Kroon F, et al. EULAR provisional recommendations for the management of rheumatic and musculoskeletal diseases in the context of SARS CoV-2. Ann Rheum Dis 2020;79:851-8.

2 Bijlsma JWJ. EULAR December 2020 viewpoints on SARS-CoV-2 vaccination in patients with RMDs. Ann Rheum Dis 2021;80:411-2.

3 van der Heijde D, Aletaha D, Carmona L, et al. 2014 update of the EULAR standardised operating procedures for EULAR-endorsed recommendations. Ann Rheum Dis 2015;74:8-13.

4 Kroon FPB, Najm A, Alunno A, et al. Risk and prognosis of SARS-CoV-2 infection and vaccination against SARS-CoV-2 in rheumatic and musculoskeletal diseases: a systematic literature review to inform EULAR recommendations. Ann Rheum Dis 2021. doi:10.1136/annrheumdis-2021-221575. [Epub ahead of print: 07 Dec 2021].

5 Bower H, Frisell T, Di Giuseppe D, et al. Impact of the COVID-19 pandemic on morbidity and mortality in patients with inflammatory joint diseases and in the general population: a nationwide Swedish cohort study. Ann Rheum Dis 2021:80:1086-93.

6 Cordtz R, Lindhardsen J, Soussi BG, et al. Incidence and severeness of COVID-19 hospitalization in patients with inflammatory rheumatic disease: a nationwide cohort study from Denmark. Rheumatology 2021;60:SI59-67. 
7 Strangfeld A, Schäfer M, Gianfrancesco MA, et al. Factors associated with COVID-19related death in people with rheumatic diseases: results from the COVID-19 global rheumatology alliance physician-reported registry. Ann Rheum Dis 2021;80:930-42.

8 Schäfer M, Strangfeld A, Hyrich KL, et al. Response to: 'Correspondence on 'Factors associated with COVID-19-related death in people with rheumatic diseases: results from the COVID-19 Global Rheumatology Alliance physician reported registry" by Mulhearn et al. Ann Rheum Dis 2021. doi:10.1136/annrheumdis-2021-220134. [Epub ahead of print: 01 Mar 2021].

9 Sparks JA, Wallace ZS, Seet AM, et al. Associations of baseline use of biologic or targeted synthetic DMARDs with COVID-19 severity in rheumatoid arthritis: results from the COVID-19 global rheumatology alliance physician registry. Ann Rheum Dis 2021:80:1137-46.

10 Isaacs JD, Burmester GR. Smart battles: immunosuppression versus immunomodulation in the inflammatory RMDs. Ann Rheum Dis 2020;79:991-3.

11 Agarwal A, Rochwerg B, Lamontagne F, et al. A living who guideline on drugs for covid-19. BMJ 2020;370:m3379.

12 European Alliance of Associations for Rheumatology. Eu shortage of arthritis drugs due to use as treatment for severe CV-19 cases. Available: https://www.eular. org/sysModules/obxContent/files/www.eular.2015/1_42291DEB-50E5-49AE5726D0FAAA83A7D4/eular_press_release_eu_shortage_of_arthritis_drugs_due to_use_as_treatment_for_severe_cv_19_cases_(rev_16_09).pdf [Accessed 28 Nov 2021].

13 American College of Rheumatology. American College of rheumatology update on tocilizumab shortages. Available: https://www.rheumatology.org/About-Us/ Newsroom/Press-Releases/ID/1160 [Accessed 28 Nov 2021].

14 World Health Organization. Joint statement from Unitaid and the world Health organization (on behalf of the access to COVID-19 tools accelerator) regarding availability of tocilizumab. Available: https://www.who.int/news/item/18-08-2021joint-statement-from-unitaid-and-the-world-health-organization-(on-behalf-of-theaccess-to-covid-19-tools-accelerator)-regarding-availability-of-tocilizumab [Accessed 28 Nov 2021].

15 American College of Rheumatology. Guiding principles from the American College of rheumatology for scarce resource allocation during the COVID-19 pandemic: IL-6 inhibition, 2021. Available: https://www.rheumatology.org/Portals/0/Files/ Guiding-Principles-Scarce-Resource-Allocation-IL-6-Inhibition.pdf [Accessed 28 Nov 2021].

16 British Society for Rheumatology. COVID-19 guidance. Available: https://www. rheumatology.org.uk/practice-quality/covid-19-guidance [Accessed 28 Nov 2021].

17 European Medicines Agency. Questions and answers on COVID-19 vaccination in the EU. Available: https://ec.europa.eu/info/live-work-travel-eu/coronavirus-response/ safe-covid-19-vaccines-europeans/questions-and-answers-covid-19-vaccination-eu_ en [Accessed 28 Nov 2021].
18 Mehta P, Meeran K, Macphie E, et al. Variability in counselling for adrenal insufficiency in COVID-19 and beyond: a survey of rheumatology practice. Lancet Rheumatol 2021;3:e92-4

19 Duru N, van der Goes MC, Jacobs JWG, et al. EULAR evidence-based and consensusbased recommendations on the management of medium to high-dose glucocorticoid therapy in rheumatic diseases. Ann Rheum Dis 2013;72:1905-13.

20 Mikuls TR, Johnson SR, Fraenkel L, et al. American College of rheumatology guidance for the management of rheumatic disease in adult patients during the COVID-19 pandemic: version 3. Arthritis Rheumatol 2021;73:e1-12.

21 Alunno A, Najm A, Machado PM, et al. EULAR points to consider on pathophysiology and use of immunomodulatory therapies in COVID-19. Ann Rheum Dis 2021;80:698-706.

22 Alunno A, Najm A, Machado PM, et al. 2021 update of the EULAR points to consider on the use of immunomodulatory therapies in COVID-19. Ann Rheum Dis 2022;81:34-40.

23 Alunno A, Najm A, Mariette X, et al. Immunomodulatory therapies for the treatment of SARS-CoV-2 infection: an update of the systematic literature review to inform EULAR points to consider. RMD Open 2021;7:e001899.

24 Alunno A, Najm A, Mariette X, et al. Immunomodulatory therapies for SARS-CoV-2 infection: a systematic literature review to inform EULAR points to consider. Ann Rheum Dis 2021:80:803-15.

25 Furer V, Rondaan C, Heijstek MW, et al. 2019 update of EULAR recommendations for vaccination in adult patients with autoimmune inflammatory rheumatic diseases. Ann Rheum Dis 2020;79:39-52.

26 Curtis JR, Johnson SR, Anthony DD, et al. American College of rheumatology guidance for COVID-19 vaccination in patients with rheumatic and musculoskeletal diseases: version 3. Arthritis Rheumatol 2021;73:e60-75.

27 Bar-On YM, Goldberg Y, Mandel M, et al. Protection of BNT162b2 vaccine booster against Covid-19 in Israel. N Eng/J Med 2021;385:1393-400.

28 Patalon T, Gazit S, Pitzer VE, et al. Odds of testing positive for SARS-CoV-2 following receipt of 3 vs 2 doses of the BNT162b2 mRNA vaccine. JAMA Intern Med 2021. doi:10.1001/jamainternmed.2021.7382. [Epub ahead of print: 30 Nov 2021].

29 Mikuls TR, Johnson SR, Fraenkel L, et al. American College of rheumatology guidance for the management of rheumatic disease in adult patients during the COVID-19 pandemic: version 1. Arthritis Rheumatol 2020;72:1241-51.

30 National Institute for Health and Care Excellence. COVID-19 rapid guideline: rheumatological autoimmune, inflammatory and metabolic bone disorders. Available: https://www.nice.org.uk/guidance/ng 167 [Accessed 28 Nov 2021].

31 American College of Rheumatology. COVID-19 vaccine clinical guidance summary for patients with rheumatic and musculoskeletal diseases (version 4), 2021. Available: https://www.rheumatology.org/Portals/0/Files/COVID-19-Vaccine-Clinical-GuidanceRheumatic-Diseases-Summary.pdf [Accessed 28 Nov 2021]. 